\title{
Deuterium release from implanted beryllium and beryllium oxide
}

\author{
M. Oberkofler ${ }^{\mathrm{a}}$, Ch. Linsmeier ${ }^{\mathrm{a}, *}$ \\ ${ }^{a}$ Max-Planck-Institut für Plasmaphysik, EURATOM Association, \\ Boltzmannstr. 2, 85748 Garching b. München, Germany
}

\begin{abstract}
The retention and release of deuterium is compared for clean metallic beryllium and beryllium oxide. Deuterium energies of $600 \mathrm{eV}$ per atom are applied and implantation fluences are above the threshold for supersaturation. Desorption experiments subsequent to implantation into metallic Be are extrapolated to a perfectly homogeneous implantation spot. It is assessed that more than $80 \%$ of the D retained after implantation with homogeneous high fluences are released below $500 \mathrm{~K}$. All retained D is released below $800 \mathrm{~K}$. The oxide layer is grown by stepwise oxidation of Be in oxygen atmosphere and characterized by Rutherford backscattering spectrometry. The oxide thickness is large enough to guarantee deuterium implantation completely within the oxide layer. The release of deuterium from $\mathrm{BeO}$ proceeds at a slow rate over the whole temperature range from 350 to $850 \mathrm{~K}$. At this temperature only $75 \%$ of the amount retained after implantation has desorbed.
\end{abstract}

Keywords:

JNM: B0100, I0400, O0200, H0400, F0400

PSI-19: Implantation, Beryllium, Deuterium inventory, Oxygen, Outgassing

PACS: 28.52.Fa, 68.43.Vx, 82.20.Pm, 61.72.Ww

\footnotetext{
*Corresponding author

Email address: linsmeier@ipp.mpg.de (Ch. Linsmeier )
} 


\section{Introduction}

Beryllium is planned as a plasma facing material in the international fusion experiment ITER [1]. According to the currently foreseen design the main chamber with an area of $700 \mathrm{~m}^{2}$ will be covered by Be. The retention and release behavior of plasma facing materials with respect to hydrogen isotopes is an issue in such an experiment as well as in a future reactor due to the radioactivity of the isotope tritium that is a fuel in the fusion reaction. Consequently, the amount of hydrogen isotopes retained after implantation into Be and the temperatures where it is released have been the subject of many experimental investigations (for reviews see [2] and [3]).

The fraction of the incident hydrogen that is retained in single or polycrystalline Be after implantation has been shown to saturate with increasing fluence. The release spectra also feature a strong fluence dependence $[4,5]$ : After implantation of $\mathrm{D}$ at an energy of $1 \mathrm{keV}$ per atom to fluences below $1 \times 10^{21} \mathrm{~m}^{-2}$ the release in a subsequent desorption experiment occurs in the temperature range between 700 and $900 \mathrm{~K}$. At higher fluences a narrow release regime appears around $450 \mathrm{~K}$. In ITER the fluences expected to impinge onto the Be first wall are on the order of $1 \times 10^{22} \mathrm{D} \mathrm{m}^{-2}$ per discharge or higher [3]. The foreseen annealing temperature of the first wall is $500 \mathrm{~K}$ [6]. Section 3 is dedicated to the efficiency of this annealing temperature in removing D after implantation to high fluences.

In the presence of oxygen atoms or molecules Be is highly reactive towards oxidation [7]. At room temperature an oxide layer of a few $\mathrm{nm}$ is self-passivating and inhibits further growth. At elevated temperatures oxide layers of several tens of $\mathrm{nm}$ can be grown in oxygen atmosphere. Diffusion of Be atoms from the interface through the oxide to the surface has been identified to be the rate limiting mechanism for this thermal oxide layer growth [8].

On one hand the strong tendency of Be to oxidize is advantageous in 
ITER. Gettering of oxygen by the Be reduces the amount of residual impurities in the core plasma, which in turn leads to desired lower radiative losses. On the other hand it opens questions on how an oxide layer affects hydrogen isotope retention in Be (by forming a permeation barrier for outdiffusing hydrogen) and to what extent hydrogen isotopes retained in thick $\mathrm{BeO}$ layers are an issue. In a previous report it was concluded that the influence of a $3 \mathrm{~nm}$ thick oxide layer on the release behavior of deuterium implanted at $\mathrm{keV}$ energies into Be is small and not rate limiting [9]. It was argued that this is due to the $\mathrm{D}$ atoms being trapped in the metallic $\mathrm{Be}$ bulk after implantation and that the release from these traps dominantly governs the desorption rate. The retention of $\mathrm{D}$ when implanted into $\mathrm{BeO}$ can be qualitatively different. Possible formation of beryllium hydroxide within the bulk of the material may alter the desorption behavior [10, 11]. This is investigated in section 5 .

\section{Experimental procedures}

Most of the experiments presented here were performed at the experimental apparatus ARTOSS at IPP [12]. We focus in this section on the experimental details and conditions relevant for the specific experiments reported on. A more detailed description of the general experimental procedures at the ARTOSS apparatus can be found in [4].

The Be samples used are polycrystalline square plates with $10 \mathrm{~mm}$ side length and $0.5 \mathrm{~mm}$ thickness delivered by MaTecK GmbH. They are produced by vacuum hot isostatic pressing of Be powder and subsequent hot rolling. Polishing with diamond pads and diamond spray results in specular surfaces with no visible scrub marks and a roughness amplitude $R_{a}$ below

$10 \mathrm{~nm}$. The bulk purity of these samples is greater than $99.0 \%$. For the experiments reported in section 3 the surface is cleaned in vacuo by cyclic bombardment with argon and subsequent annealing in order to reduce the 
oxygen contamination of the surface to a few monolayers of $\mathrm{BeO}$ as estimated from X-ray photoelectron spectroscopy (XPS). After annealing a silicon signal corresponding to roughly one monolayer can also be detected on the surface. This Si probably originates from the polishing process, segregates on the surface during annealing and is sputtered off during irradiation with Ar or D.

Formation and characterization of the thick oxide layers is described in section 4. Scanning electron microscopy of the oxidized surfaces is performed in a XL30 environmental scanning electron microscope from FEI.

Both the metallic and the heavily oxidized surfaces were implanted at room temperature by a mass separated beam of $\mathrm{D}_{3}^{+}$ions. The acceleration voltage was $1.8 \mathrm{kV}$ which - upon dissociation of the molecules at the sample surface - yields an implantation energy per atom of $600 \mathrm{eV}$. The implantation spot is optimized with respect to previous investigations: By means of an ion optical system the beam is focused to obtain a full-widthat-half-maximum intensity of about $1 \mathrm{~mm}$. A well-defined, homogeneous implantation area with sharp borders is achieved using a motorized sample stage and moving the sample relative to the fixed ion beam. This approach leads to local D ion fluxes in the center of the beam spot on the order of $1 \times 10^{18} \mathrm{D} \mathrm{m}^{-2} \mathrm{~s}^{-1}$, as measured with a Faraday cup of $0.5 \mathrm{~mm}$ in diameter. The D fluences given in the caption of figure 2 are the accumulated homogeneous fluences over the implanted rectangle. The insert in figure 2 shows a photograph of the implantation spot after bombardment of an amorphous hydrocarbon layer on top of a silicon substrate by D. The homogeneously implanted area $(A)$ is surrounded by a region $(B)$ of about $0.8 \mathrm{~mm}$ in width which was implanted to a reduced fluence.

After implantation with D the Be samples are heated up with a linear temperature ramp of $0.7 \mathrm{~K} \mathrm{~s}^{-1}$ in line-of-sight of a quadrupole mass spectrometer (QMS). The ramping starts at room temperature and stops 
at $1000 \mathrm{~K}$ for the metallic Be and at $850 \mathrm{~K}$ for the heavily oxidized Be. The latter temperature was chosen in order not to activate the Be diffusion through the oxide layer which would lead to disintegration of the layer. The temperature-programmed desorption (TPD) spectra plotted in figure 2 show the calibrated QMS signals for the mass to charge ratio of $m / q=4$, corresponding to molecular $\mathrm{D}_{2}^{+}$. The signals for mass to charge ratios corresponding to other $\mathrm{D}$ containing species such as $\mathrm{HD}^{+}$and $\mathrm{HDO}^{+}$remain at least one order of magnitude below the $\mathrm{D}_{2}^{+}$signal at any temperature.

Rutherford backscattering spectrometry (RBS) with ${ }^{4} \mathrm{He}$ at an energy of $1.2 \mathrm{MeV}$ is employed to gain information on the absolute amount and the depth profile of the thermally grown oxide. The amount of D retained after implantation is determined by nuclear reaction analysis (NRA) with $550 \mathrm{keV}{ }^{3} \mathrm{He}$ detecting the protons that result from the reaction $\mathrm{D}\left({ }^{3} \mathrm{He}, \mathrm{p}\right) \alpha$.

\section{Release behavior at high fluences}

Upon implantation of metallic Be with $\mathrm{D}$, the $\mathrm{D}$ is trapped in the implantation range. At high fluences saturation occurs within this range as discussed in [4]. The depth over which the implanted D atoms are distributed depends on the implantation energy per atom [13]. A smaller implantation energy leads to implantation closer to the surface. Accumulation of D in a thinner layer must consequently lead to saturation at a lower fluence compared to implantation at higher energies. In figure 1 the retained amount is plotted against the implanted fluence. The open (blue) circles are data taken from [4] and correspond to implantation of $\mathrm{D}$ at $1 \mathrm{keV}$ per atom into single crystalline Be. (These data are taken for comparison because it was shown in [13] that the threshold fluence for saturation is not influenced by the presence of grain boundaries in polycrystalline Be.) Up to fluences on the order of $1 \times 10^{21} \mathrm{~m}^{-2}$ the experimental results lie close to the line in-

dicating $100 \%$ retention. At higher fluences saturation causes a deviation 
from this line towards lower retained fractions. The squares and the triangle represent measurements of the retained amount after implantation into polycrystalline Be at 400 and at $600 \mathrm{eV}$, respectively. As expected, the retained fraction shows a saturation behavior already at lower fluences. At the highest measured fluence of $2.8 \times 10^{21} \mathrm{~m}^{-2}$ the retained areal density lies below the one for $1 \mathrm{keV}$ implantation by a factor of 2 .

The light-colored (green) line in figure 2 shows the desorption spectrum after implantation to this fluence. The total retained areal density (calculated from the time integral of the spectrum) amounts to $8.6 \times 10^{20} \mathrm{D} \mathrm{m}^{-2}$ which is $30 \%$ of the implanted fluence. The observed desorption in a low temperature $(\mathrm{LT})$ and a high temperature $(\mathrm{HT})$ regime is typical for Be implanted to fluences above the saturation threshold (c.f. section 1). The LT peak features a shoulder on the high temperature side which, however, is an artifact due to an observed fluctuation in the heating power. Integration over time of the spectrum below and above $500 \mathrm{~K}$ gives the areal densities released in each temperature regime. The fractions are 0.6 and 0.4 , for the LT and the HT regimes, respectively.

The D desorbing in the LT regime comes from locally supersaturated zones in the implanted volume. With increasing D fluence the Be lattice first saturates at the depth where the implantation depth profile has its maximum. Upon implantation to still higher fluences the width of the saturated zone increases. Such supersaturation occurs over the whole homogeneously implanted area $A$ indicated in the insert of figure 2. The rim area $B$ of the implantation spot is implanted to lower fluences (c.f. section 2). If this fluence is below saturation, then $\mathrm{D}$ desorbing from the area $B$ will contribute solely to the HT regime of the desorption spectrum. To extrapolate the fractions of D desorbing in the respective LT and the HT regimes to an ideal implantation spot with perfectly sharp boarders (i.e. $A / B \rightarrow \infty$ ), we assume the rim area $B$ to be implanted with half the fluence of the homoge- 
neous area $A$ and not to contain any supersaturated zones. We can estimate the amount $N_{B}$ released from $B$ by multiplying the total released amount $N_{\text {tot }}$ with the ratio of areas:

$$
N_{B}=N_{t o t} \times \frac{B}{2(A+B)}
$$

The factor $1 / 2$ comes from the additional assumption that the areal density retained in $B$ is half the areal density retained in $A$. This is consistent with the equivalent assumption for the fluences. The D trapped in the rim area contributes only to the HT regime of the desorption spectrum. We consequently calculate the fraction that would be released in the HT region for perfectly homogeneous implantation by

$$
f_{H T}^{\text {homog }}=\frac{N_{H T}-N_{B}}{N_{t o t}} .
$$

For perfectly homogeneous implantation $N_{B}$ would not contribute at all to the desorption spectrum as there would be no rim area implanted to lower fluences. Therefore, the D fraction released in the LT regime for the ideal case amounts to

$$
f_{L T}^{h o m o g}=1-f_{H T}^{\text {homog }} .
$$

The implanted area for the light-coloured (green) spectrum in figure 2 was $2.8 \times 2.2 \mathrm{~mm}^{2}$. From the photograph in the insert the boarder region is estimated to be about $0.8 \mathrm{~mm}$ wide. The areas $A$ and $B$ are calculated from these values and inserted into the the above equations together with $N_{t o t}$ and $N_{H T}$ which are, respectively, the total integral and the integral above $500 \mathrm{~K}$ of the desorption spectrum in figure 2. The resulting idealized fractions released in the LT and the HT regimes are

$$
f_{L T}^{\text {homog }}=0.87 \quad \text { and } \quad f_{H T}^{\text {homog }}=0.13
$$

The value of $0.8 \mathrm{~mm}$ taken for the width of the boarder region $B$ is rather conservative. Therefore this extrapolation gives a lower limit for the 
fraction of retained D to be released in the LT regime after homogeneous implantation of large areas of Be to fluences far above the saturation threshold.

\section{Growth and characterization of BeO layers}

Oxidation of the Be surfaces was induced by heating the Be samples in an oven under pure oxygen at ambient pressure. Several samples were heated stepwise to temperatures up to $890 \mathrm{~K}$ with holding times between 1 and 10 hours. The oxide layer thicknesses were measured by RBS after each temperature step. As pointed out in the introduction it is known that the growth mechanism of thick oxide layers on Be is the diffusion of Be atoms through the oxide layer. As deduced in [8] the temperature-dependent rate coefficient $k$ for the layer growth can be calculated from the holding time $t$ and the thickness increase $x-x_{0}$ at each temperature by

$$
k(T)=\frac{x^{2}-x_{0}^{2}}{t} \propto D(T) .
$$

This reaction rate coefficient is proportional to the diffusion coefficient $D$ for diffusion of $\mathrm{Be}$ in $\mathrm{BeO}$. From the variation of the rate coefficient with temperature we can obtain a value for the activation barrier $E_{a c t}$ in the Arrhenius form of the diffusion coefficient:

$$
D(T)=D_{0} \exp \frac{-E_{a c t}}{k_{B} T}
$$

It is observed that the rate coefficient is lower for samples heated for 11 hours than for samples heated for just 1 hour. This indicates that after long heating times the oxide layer growth does not follow a square root dependence on time anymore. Instead, the oxidation slows down more quickly and probably eventually stops. Therefore, in order to estimate the activation barrier $E_{a c t}$ only thickness measurements after heating steps of 1 hour duration were taken into account. 
In figure 3 the logarithm of $k$ is plotted against the inverse temperature. The number in brackets indicate the layer thickness before and after each heating step (in $10^{20}$ atoms $\mathrm{m}^{-2}$ ). An initial thickness above $5 \times 10^{20}$ atoms

$\mathrm{m}^{-2}$ indicates that the sample has undergone preceding heating steps. For the reason mentioned above those data are not included when the heating duration exceeded 1 hour. The slope of a linear fit through the data points yields an activation energy of $0.8 \mathrm{eV}$ for the diffusion of $\mathrm{Be}$ in $\mathrm{BeO}$. This value is in good agreement with the $0.73 \pm 0.14 \mathrm{eV}$ obtained in [8]. However, the scatter in the calculated rate coefficients is considerable. The growth rate seems to be influenced by the layer thickness at the onset of the heating step or by the temperature history.

SEM micrographs of the oxidized surfaces reveal adherent oxide layers with nm sized pores and cracks, presumably running along grain boundaries. Oxygen depth profiles measured by RBS are simulated by SIMNRA [14, 15]. These profiles are laterally averaged over the ${ }^{4} \mathrm{He}$ ion beam diameter, i.e. over about $1 \mathrm{~mm}^{2}$. On the sample used for implantation with D stoichiometric $\mathrm{BeO}$ is only observed in the first $20 \mathrm{~nm}$ below the surface. Then the oxygen concentration decreases towards 0.33 at a depth of about $60 \mathrm{~nm}$ and falls to the intrinsic amount of less than 0.01 around $80 \mathrm{~nm}$.

\section{Deuterium retention in $\mathrm{BeO}$}

According to static simulations with SDTrim.SP $[16,17]$ the maximum of the implantation depth profile after irradiation of $\mathrm{BeO}$ with $\mathrm{D}$ at $600 \mathrm{eV}$ lies about $20 \mathrm{~nm}$ below the surface. This depth is less than the thickness of the oxygen-rich layer. Therefore at this energy most of the D is implanted into the layer and not beyond it.

The full red data point in figure 1 corresponds to the retained amount as measured by NRA after implantation of $2.8 \times 10^{21} \mathrm{D} \mathrm{m}^{-2}$. It lies close to the point for implantation of metallic Be at this energy up to a similar 
fluence. According to this measurement, the retention behavior is similar for the two cases.

However, the release behaviors are totally different: It can be seen in figure 2 that the LT desorption stage, which is pronounced in the case of metallic Be, is not observed at all upon desorption from BeO. Instead, release of $\mathrm{D}$ is observed at a comparatively low rate over the whole temperature range from 350 to $850 \mathrm{~K}$. NRA measurements after cooling down of the sample show that only about $75 \%$ of the implanted D have been released upon heating to $850 \mathrm{~K}$.

\section{Conclusion}

More than $80 \%$ of the D retained after room temperature implantation at $600 \mathrm{eV}$ to high fluences into polycrystalline Be is released below the baking temperature of $500 \mathrm{~K}$ foreseen for the ITER first wall. However, care must be taken when drawing conclusions for ITER from this result as it might be modified when implantation occurs at elevated temperatures [9].

RBS analysis of the thermal oxidation of Be in oxygen atmosphere yields an activation barrier of $0.8 \mathrm{eV}$ for the diffusion of $\mathrm{Be}$ in $\mathrm{BeO}$. This value is in good agreement with a previous result.

The retained fraction of $\mathrm{D}$ implanted into $\mathrm{BeO}$ at $600 \mathrm{eV}$ to a fluence above the saturation threshold is similar to the one for polycrystalline metallic Be. The desorption characteristics, however, are different. Instead of dominant release around $450 \mathrm{~K}$, desorption at a low rate is observed in the whole temperature range up to $850 \mathrm{~K}$. At this temperature $25 \%$ of the amount retained after implantation is still present in the sample, while all retained $\mathrm{D}$ is released below $800 \mathrm{~K}$ from the metallic Be. This shows that the efficiency of the $500 \mathrm{~K}$ baking temperature to reduce the retained amount of implanted D is dramatically reduced in the case of thick oxide layers forming on the beryllium first wall. Whether such thick oxide layers will form 
in ITER depends on the amount of oxygen impurities present, as well as on the temperature of the Be surface and on the impinging fluxes of energetic ions that will cause thinning of the layer by sputtering. 


\section{References}

[1] M. Shimada, D.J. Campbell, V. Mukhovatov, M. Fujiwara, N. Kirneva, K. Lackner, M. Nagami, V.D. Pustovitov, N. Uckan, J. Wesley, N. Asakura, A.E. Costley, A.J.H. Donné, E.J. Doyle, A. Fasoli, C. Gormezano, Y. Gribov, O. Gruber, T.C. Hender, W. Houlberg, S. Ide, Y. Kamada, A. Leonard, B. Lipschultz, A. Loarte, K. Miyamoto, V. Mukhovatov, T.H. Osborne, A. Polevoi, and A.C.C. Sips, Nucl. Fusion 47 (2007) S1.

[2] R. A. Anderl, R. A. Causey, J. W. Davis, R. P. Doerner, G. Federici, A. A. Haasz, G. R. Longhurst, W. R. Wampler, and K. L. Wilson, J. Nucl. Mater. 273 (1999) 1.

[3] J. Roth, E. Tsitrone, A. Loarte, Th. Loarer, G. Counsell, R. Neu, V. Philipps, S. Brezinsek, M. Lehnen, P. Coad, Ch. Grisolia, K. Schmid, K. Krieger, A. Kallenbach, B. Lipschultz, R. Doerner, R. Causey, V. Alimov, W. Shu, O. Ogorodnikova, A. Kirschner, G. Federici, and A. Kukushkin, J. Nucl. Mater. 390-391 (2009) 1.

[4] M. Reinelt, A. Allouche, M. Oberkofler, and Ch. Linsmeier, New J. Phys. 11 (2009) 043023.

[5] M. Oberkofler, M. Reinelt, S. Lindig, and Ch. Linsmeier, Nucl. Instrum. Meth. B 267 (2009) 718.

[6] R.A. Pitts, A. Kukushkin, A. Loarte, A. Martin, M. Merola, C.E. Kessel, V. Komarov, and M. Shimada, Phys. Scr. T138 (2009) 014001.

[7] Ch. Linsmeier and J. Wanner, Surf. Sci. 454-456 (2000) 305.

[8] J. Roth, W. R. Wampler, and W. Jacob, J. Nucl. Mater. 250 (1997) 23.

[9] M. Reinelt and Ch. Linsmeier, J. Nucl. Mater. 390-391 (2009) 568. 
[10] A. Markin, A. Gorodetsky, F. Scaffidi-Argentina, H. Werle, C.H. Wu, and A. Zakharov, Fusion Technol. 38 (2000) 363.

[11] S. Zalkind, M. Polak, and N. Shamir, Surf. Sci. 385 (1997) 318.

[12] Ch. Linsmeier, P. Goldstraß, and K.U. Klages, Phys. Scr. T94 (2001) 28.

[13] M. Oberkofler, M. Reinelt, A. Allouche, S. Lindig, and Ch. Linsmeier, Phys. Scr. T138 (2009) 014036.

[14] M. Mayer, SIMNRA user's guide, Report IPP 9/113, Max-PlanckInstitut für Plasmaphysik, Germany (1997).

[15] M. Mayer, The fifteenth international conference on the application of accelerators in research and industry 475 (1999) 541.

[16] W. Eckstein, Computer Simulation of Ion-Solid Interactions, volume 10 (Springer, Berlin, Series in Materials Science, 1991).

[17] W. Eckstein, R. Dohmen, A. Mutzke, and R. Schneider, SDTRIM.SP: A Monte Carlo Code for Calculating Collision Phenomena in Randomized Targets, Report IPP 12/3, Max-Planck-Institut für Plasmaphysik, Germany (2007). 
Figure 1: Retained areal density of D as a function of irradiated D fluence for implantation at various energies into single crystalline $\mathrm{Be}$, polycrystalline $\mathrm{Be}$ and $\mathrm{BeO}$.

Figure 2: Desorption flux of $\mathrm{D}_{2}(m / q=4 \mathrm{amu} / \mathrm{e})$ as a function of the sample surface temperature, recorded during annealing of polycrystalline $\mathrm{Be}$ and $\mathrm{BeO}$ previously implanted at room temperature with $600 \mathrm{eV} \mathrm{D}$ to fluences of $2.8 \times 10^{21} \mathrm{~m}^{-2}$ and $2.4 \times 10^{21} \mathrm{~m}^{-2}$, respectively. The heating rate is $0.7 \mathrm{~K} / \mathrm{s}$. The insert shows the implantation spot after irradiation of an a-C:H layer on Si. The homogeneous implantation area $A$ and the rim area $B$ can be clearly distinguished. The red line indicates the baking temperature of $500 \mathrm{~K}$ foreseen for ITER.

Figure 3: Logarithm of the reaction rate coefficient for thermal oxidation of various Be samples versus temperature. The holding time at each temperature was 1 hour. The numbers in brackets indicate the BeO layer thickness (in $10^{20}$ atoms $\mathrm{m}^{-2}$ ) before and after each heating step. 


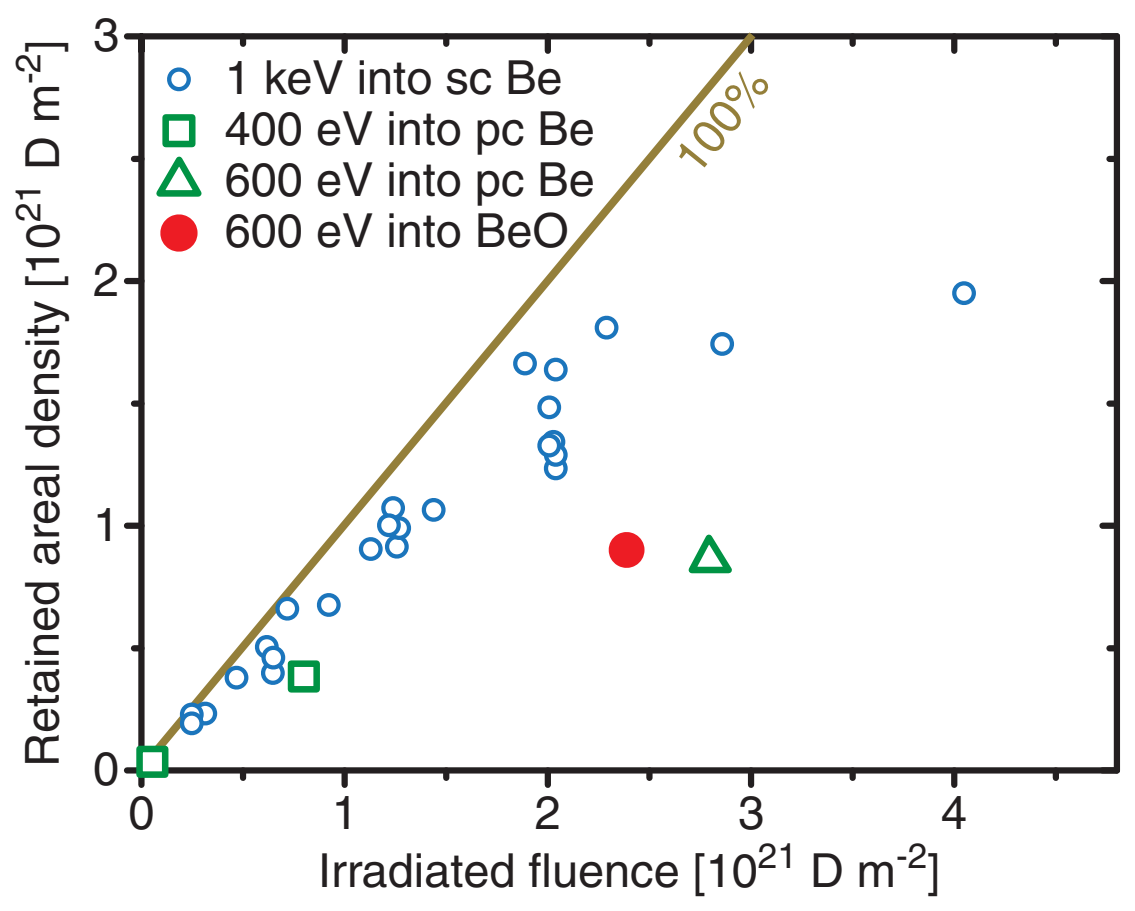

Fig. 1 (Oberkofler and Linsmeier) 


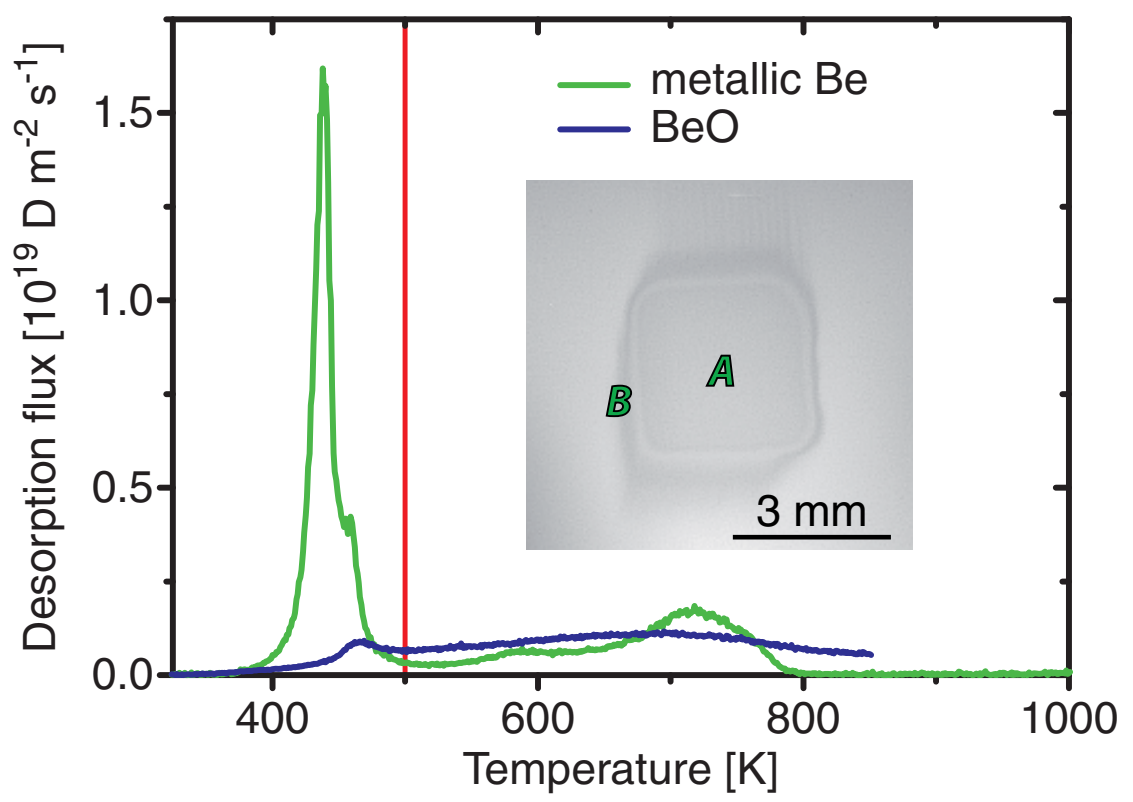

Fig. 2 (Oberkofler and Linsmeier) 


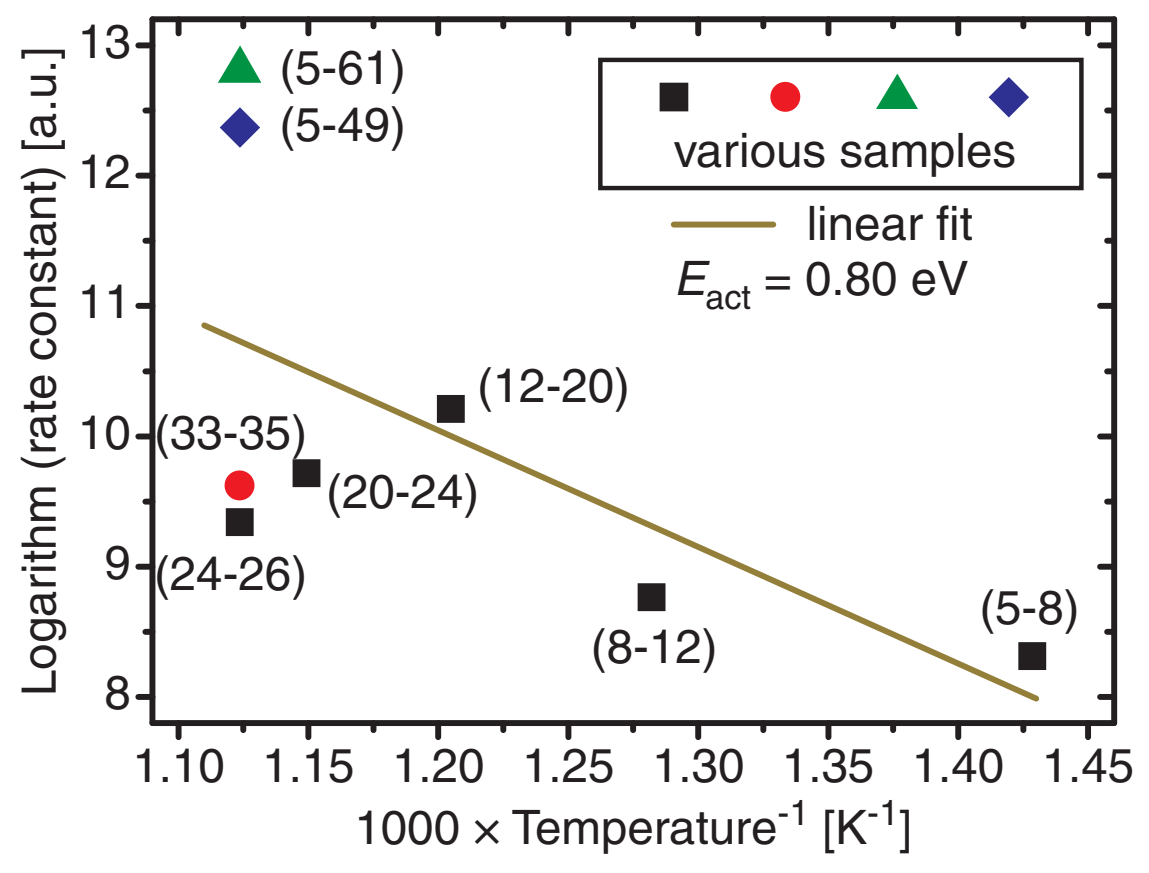

Fig. 3 (Oberkofler and Linsmeier) 